\title{
Generation of Broadband VUV Light Using Third-Order Cascaded Processes
}

\author{
L. Misoguti, S. Backus, C. G. Durfee, ${ }^{*}$ R. Bartels, M. M. Murnane, ${ }^{\dagger}$ and H. C. Kapteyn \\ JILA and Department of Physics, University of Colorado at Boulder, Boulder, Colorado 80309-0440
}

(Received 2 August 2000; revised manuscript received 1 February 2001; published 14 June 2001)

\begin{abstract}
We report the first demonstration of broadband VUV light generation through cascaded nonlinear wave mixing in a gas. Using a hollow-fiber geometry to achieve broad-bandwidth phase-matching, frequency conversion of ultrashort-pulse Ti:sapphire laser pulses from the visible into the deep UV around 200 and $160 \mathrm{~nm}$ is achieved. A new type of quasi-phase-matching is also observed in the VUV for the first time. Conversion using cascaded processes exhibits higher efficiencies, shorter pulse durations, and broader bandwidths than other schemes for generating light in the deep UV, and will enable many applications in science and technology.
\end{abstract}

DOI: $10.1103 /$ PhysRevLett.87.013601

Nonlinear optical techniques have been used extensively to extend the wavelength range of coherent light sources, making it possible to efficiently generate light in the visible and ultraviolet (UV) regions of the spectrum. In this Letter, we describe a new approach in nonlinear optics for the generation of ultra-short-pulse, short-wavelength light. In past work using a hollow-waveguide geometry for frequency conversion [1-3], we demonstrated that it is possible to achieve high efficiency conversion into the UV and soft-x-ray regions of the spectrum. In this Letter, we show that this high efficiency allows us to drive a cascaded nonlinear up-conversion process from the visible to the VUV region of the spectrum with macroscopic conversion efficiencies. Moreover, these cascaded processes are more efficient and generate broader bandwidths than past schemes, and are therefore ideal for ultra-short-pulse conversion into the VUV. Finally, we also observe a type of quasi-phase-matching in the deep-UV region of the spectrum for the first time. Cascaded four-wave mixing processes up-convert light in large $(1.5 \mathrm{eV})$ energy steps (unlike processes such as anti-Stokes Raman), and are therefore uniquely suited for generating intense light in the range of photon energy $5-15 \mathrm{eV}$. This is a region of great interest for studies of small molecules, chemistry, material science, and lithography [4-6]. Chemical-physics studies, in particular, may benefit tremendously from the ability to excite shaped "wave packets" in deep-UV absorbing molecules, for applications in coherent control of molecular reactions $[7,8]$.

Past work has demonstrated conversion efficiencies higher than $21 \%$ using nonlinear crystals to convert pulses to $208 \mathrm{~nm}$ [9]. However, present solid-state materials are transparent only at wavelengths longer than $\sim 160-$ $210 \mathrm{~nm}$, and simple phase-matched harmonic generation is possible only to wavelengths of $\sim 210 \mathrm{~nm}$ [10]. Furthermore, the group velocity walk-off between the fundamental and harmonic severely limits the bandwidth and pulse width in the UV [11]. Phase-matched VUV frequency conversion in gases has been achieved in the past by exploiting the negative dispersion which exists for light at wavelengths just shorter than a resonant absorption line
PACS numbers: 42.50.Gy, 42.65.Ky, 42.65.Wi, 42.81.Qb

[12-14]. However, this near-resonant requirement limits the phase-matching bandwidth, tunability, pulse width, and conversion efficiency. Electromagnetically induced transparency can also be used to obtain extremely efficient conversion of long-pulse ultraviolet light to shorter wavelengths - from 233 to $186 \mathrm{~nm}$ with near-unity efficiency [15], and into the VUV with more modest efficiencies [16]. Cascaded second-order nonlinear processes have also previously been studied as a means to obtain an enhanced effective third-order nonlinearity $[17,18]$. However, third-order cascaded nonlinear processes [19,20] have not been as extensively studied, with the exception of anti-Stokes Raman scattering, which can be used for generating deep-UV light with modest conversion efficiency [21]. In recent work, Crespo et al. [22] have successfully used the third-order cascaded process to extend frequency generation in solid state materials from $400-750 \mathrm{~nm}$ and pulsewidths of $40 \mathrm{fs}$. Cascaded frequency mixing in gases has also recently been discussed in a theoretical framework [23].

For our work, we used a gas-filled hollow-core fiber as a phase-matching medium [1]. In addition to significantly increasing the interaction length, thereby compensating for the low gas density, the guided-mode geometry allows phase-matched frequency conversion. For a waveguide of diameter $a$, the total wave vector for light at wavelength $\lambda$ is given by

$$
k(\lambda) \approx \frac{2 \pi}{\lambda}+\frac{2 \pi P \delta(\lambda)}{\lambda}-\frac{u_{n m}^{2} \lambda}{4 \pi a^{2}},
$$

where $P$ is the pressure of the gas, $\delta(\lambda)$ is related to the index of refraction of the gas by $n=1+P \delta(\lambda)$, and $u_{n m}$ is a discrete constant that varies with the propagation mode in the fiber. The three terms in this expression correspond to the contribution from free space propagation, gas dispersion, and the waveguide dispersion, respectively. For a particular nonlinear mixing process, the net phase mismatch $(\Delta k)$ is given by the vector sum of all waves involved, where the wave vector for each wave is given by Eq. (1). By adjusting the gas pressure, gas species, waveguide size, and spatial mode in which the light propagates, the phase mismatch can be tuned to achieve the phase-matching 
condition of $\Delta k=m k_{\text {pump }}-q k_{\text {idler }}-k_{\text {signal }}=0$, where $m$ and $q$ are the numbers of photons involved.

The experimental setup is shown in Fig. 1. Pulses at a repetition rate of $1 \mathrm{kHz}$, with an energy of $1 \mathrm{~mJ}$, and duration $25 \mathrm{fs}$, are generated in a Ti:sapphire amplifier system [24]. A beam splitter directs $70 \%$ of the energy through a $250 \mu \mathrm{m}$ thick beta-barium borate doubling crystal to generate the pump light at frequency $2 \omega(400 \mathrm{~nm})$, which is then coupled into the hollow-core fiber of $125 \mu \mathrm{m}$ inside diameter. The other $30 \%$ of the laser output is focused directly into the hollow-core fiber, to serve as the idler at frequency $\omega(800 \mathrm{~nm})$. The hollow-core fiber is a fused silica capillary mounted in a cell that can be evacuated and filled with gas, typically Ar. The generated $4 \omega$ and $5 \omega$ light is spectrally resolved onto a phosphor-coated window, and detected using a charge-coupled device camera.

Light at $267 \mathrm{~nm}(3 \omega)$ is generated in the capillary using a difference-frequency four-wave mixing process (DFFWM) in argon: $3 \omega=2 \omega+2 \omega-\omega$. This DFFWM process is very efficient, with about $30 \%-40 \%$ of the pump light at $2 \omega$ converted to $3 \omega$. Careful mode matching and attention to the straightness has increased the efficiency by $50 \%$ over previous work [1]. Figure 2(a) shows the $3 \omega$ signal as a function of Ar pressure for a long $(80 \mathrm{~cm})$ and a short $(10 \mathrm{~cm})$ fiber. This process phase matches to the $\mathrm{EH}_{11}$ mode at pressures around 95 Torr [peak (3)]. For pulse energies of $\sim 25 \mu \mathrm{J}$ at $2 \omega$ and $\sim 30 \mu \mathrm{J}$ at $\omega$, energies between 2.5 and $10 \mu \mathrm{J}$ were generated at $3 \omega$ in the lowest $\mathrm{EH}_{11}$ mode, depending on the fiber length. With such high conversion efficiency, the intensity of the $3 \omega$ light is comparable to that of the input light. This is key to making efficient cascaded conversion to still-shorter wavelengths possible.

The $267 \mathrm{~nm}(3 \omega)$ light from the first DFFWM process serves as the seed for a second four-wave mixing step, giving rise to light at either $4 \omega$ or $5 \omega$. This second wave-mixing process can be optimized either in the same fiber or in a second, separately optimized, fiber. The output at $4 \omega(200 \mathrm{~nm})$ and $5 \omega(160 \mathrm{~nm})$ was monitored as a function of pressure, as shown in Figs. 2(b) and 2(c), for both short and long fibers. There are several processes that can result in each output; these are each distinguished experimentally by the phase-matching pressures, the output spatial modes, and the order of nonlinearity. For $4 \omega$ generation, the possible processes and their corre-



FIG. 1. Schematic diagram for cascaded guided-wave nonlinear mixing. sponding phase-matching pressures to the lowest possible spatial modes allowed are $4 \omega=2 \times 3 \omega-2 \omega$ (12 Torr, $\mathrm{EH}_{11} ; 172$ Torr, $\mathrm{EH}_{12}$ ), $4 \omega=2 \omega+3 \omega-\omega$ (40 Torr, $\left.\mathrm{EH}_{11}\right), 4 \omega=2 \times \omega+2 \omega$ (43 Torr, $\mathrm{EH}_{13}$ ), and $4 \omega=$ $3 \times 2 \omega-2 \times \omega\left(53\right.$ Torr, $\mathrm{EH}_{11} ; 129$ Torr, $\left.\mathrm{EH}_{12}\right)$. The last process is a direct fifth-order process, not a cascaded process. While each process contributes to the output signal, macroscopic conversion efficiency is expected only for those processes that are phase matched, with the highest efficiency for conversion into the lowest-order $\mathrm{EH}_{11}$ mode of the fiber. Figure 2(b) shows the $4 \omega$ signal at $200 \mathrm{~nm}$ as a function of Ar gas pressure. For short $(10 \mathrm{~cm})$ fiber lengths, phase-matched peaks are observed at pressures of 58 and 135 Torr, while, for long $(80 \mathrm{~cm})$ fibers, peaks are observed at pressures of 33, 58, 95, and 135 Torr. To identify the dominant processes contributing to the observed $4 \omega$ signal, we also investigated $4 \omega$ generation in a two-stage fiber. The pressure in the first stage was adjusted and fixed to maximize $3 \omega$ generation, while the pressure in the second stage was varied, as shown in Fig. 3. We observe a single strong phase-matched peak for $4 \omega$ generation at pressures around 33 Torr, in the lowest-order $\mathrm{EH}_{11}$ mode of the fiber. The broad peaks at high pressures are due to phase matching to high-order spatial modes in the fiber.

We believe that strong cascaded DFFWM $(4 \omega=2 \omega+$ $3 \omega-\omega)$ is the origin of peaks (1)-(3) in Fig. 2(b). Peak (1) at 33 Torr corresponds to phase matching of the $4 \omega=2 \omega+3 \omega-\omega$ DFFWM process. Peak (3)
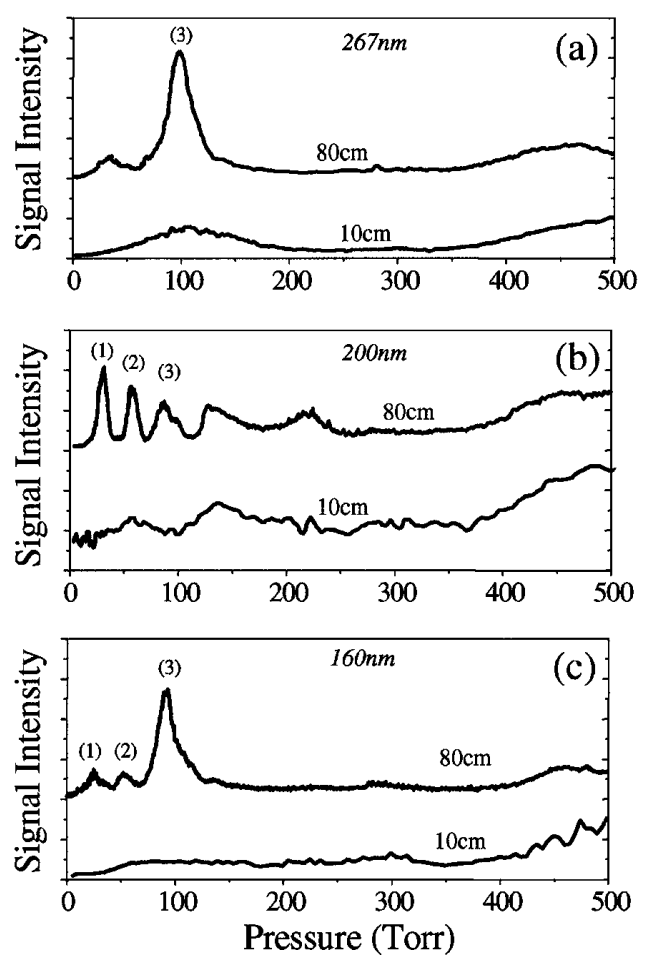

FIG. 2. Pressure tuning curves for the generation of (a) $3 \omega$ light at $267 \mathrm{~nm}$, (b) $4 \omega$ light at $200 \mathrm{~nm}$, and (c) $5 \omega$ light at $160 \mathrm{~nm}$, within a single fiber. 


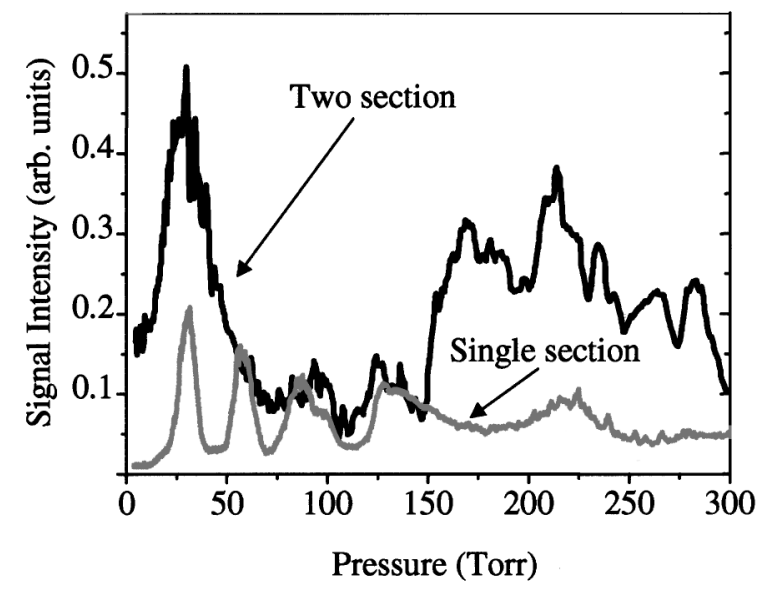

FIG. 3. Pressure tuning curves for $4 \omega$ generation in the second stage of a two-stage fiber. The pressure in the 1st stage was fixed at 100 Torr to optimize $3 \omega$ generation.

corresponds to that pressure that maximizes $3 \omega$ generation - and, hence, results in a peak in $4 \omega$ generation from the same DFFWM process-despite the fact that the DFFWM process is not phase matched. Peak (2) in the long and short fiber corresponds to quasi-phase-matching of the same process within a single fiber, as will be discussed in more detail below. Several arguments support the claim of strong cascaded DFFWM as the origin of peaks (1)-(3). First, the phase-matching pressure in the long $(80 \mathrm{~cm})$ fiber at 33 Torr is close to the predicted value of 40 Torr (The small 7 Torr discrepancy between theory and experiment is likely due to imprecise refractive index data at short wavelengths.). Second, Fig. 3 compares $4 \omega$ generation in a long one- and two-stage fiber. In the two-stage fiber, the optimum pressure for the $4 \omega=2 \omega+3 \omega-\omega$ four-wave mixing process can be adjusted independently of the $3 \omega$ generation process in the first stage. In this case, only a single pressure peak is observed for $4 \omega$ generation, at a pressure of 33 Torr, as expected for DFFWM. Third, the generated $4 \omega$ output is in the lowest-order $\mathrm{EH}_{11}$ mode of the fiber, as expected. Fourth, the observed dependence of the $4 \omega$ signal on $3 \omega$ power shown in Fig. 4 is consistent with this process. Fifth, evidence for depletion of the driving light at $3 \omega$ in the second stage of the two-stage fiber is shown in Fig. 4. The output $3 \omega$ light is observed to deplete at that pressure that optimizes $4 \omega$ generation.

Other possible processes that might give rise to the $4 \omega$ light can be ruled out. For example, the direct six-wave mixing process $(4 \omega=3 \times 2 \omega-2 \times \omega)$ that would give rise to the $4 \omega$ signal is weak because it relies on a fifthorder nonlinearity, and it should exhibit no $3 \omega$ power dependence, in contrast to Fig. 4. For sum-frequency four-wave mixing (SFFWM), although the nonlinear susceptibility is similar to DFFWM, phase matching of this process occurs only when the signal is in a high-order mode $\left(\mathrm{EH}_{13}\right.$ for $\left.4 \omega=2 \times \omega+2 \omega\right)$, which is not observed experimentally. Finally, no similar SFFWM processes are observed, even processes that should be

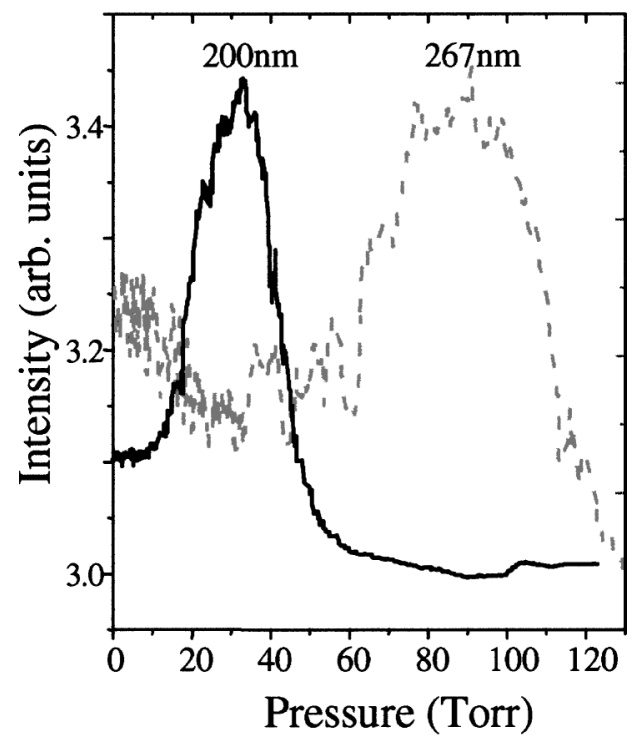

FIG. 4. Output at $3 \omega$ and $4 \omega$ as a function of pressure in the 2 nd stage of a two-stage fiber. The $3 \omega$ light depletes at that pressure that optimizes $4 \omega$ generation, demonstrating a cascaded process.

strong such as $3 \omega=\omega+\omega+\omega$, for the case of IR-only illumination.

Strong generation of light at $5 \omega$ or $160 \mathrm{~nm}$ was also observed, as can be seen from Fig. 2(c). Processes that generate phase-matched $5 \omega$ light are $5 \omega=2 \times 3 \omega-\omega$ (18 Torr, $\left.\mathrm{EH}_{11}\right), 5 \omega=4 \omega+2 \omega-\omega\left(20\right.$ Torr, $\left.\mathrm{EH}_{11}\right)$, $5 \omega=4 \omega+3 \omega-2 \omega$ (6 Torr, $\left.\mathrm{EH}_{11}\right), \quad 5 \omega=2 \omega+$ $2 \omega+\omega$ (16 Torr, $\left.\mathrm{EH}_{13}\right), 5 \omega=3 \omega+\omega+\omega$ (6 Torr, $\left.\mathrm{EH}_{13}\right)$, and $5 \omega=5 \times \omega\left(63\right.$ Torr, $\left.\mathrm{EH}_{15}\right)$. As would be expected for a cascaded process, the $5 \omega$ signal intensity is observed to follow the pressure profiles of the $3 \omega$ and $4 \omega$ signal intensities, independent of the phase-matching pressure of the $5 \omega$ process. It is also generated in the lowest-order mode of the fiber. These observations restrict the possible processes giving rise to the $5 \omega$ light to a cascaded DFFWM process: either $5 \omega=2 \times 3 \omega-\omega$, or $5 \omega=4 \omega+2 \omega-\omega$, or $5 \omega=4 \omega+3 \omega-2 \omega$.

The conversion efficiency for $4 \omega$ generation was approximately $10 \%$ of the $3 \omega$ beam, while the generated bandwidth was sufficient to support a 12 fs pulse. Crossphase modulation by the pump can produce still larger bandwidths capable of supporting sub-4-fs pulses, when higher intensities, higher pressures, and longer fibers are employed. The conversion efficiency for $5 \omega$ generation within a single fiber was approximately $0.1 \%$ or $1 \%$ of the $3 \omega$ or $4 \omega$ light, while the generated bandwidth was sufficient to support a 6 fs pulse. For both the $4 \omega$ and $5 \omega$ signals, the stability was the same as that of the laser $( \pm 2 \%)$, because we saturate the output.

Simple calculations based on Eq. (1) above can predict the phase-matching pressure, power dependence, and output modes for the various processes involved in the generation of the $4 \omega$ and $5 \omega$ light, as discussed above. However, an even more convincing argument can be made based on a model that keeps track of all the optical fields involved 
in the interaction, and predicts the output as a sum over all possible processes. We therefore developed a more sophisticated model of the generation and propagation of light at the first through fifth harmonic, where the five coupled nonlinear equations are solved for given pulse input, waveguide dimension, gas type, and pressure. The Fourier split-step method is used to model the propagation of the fields: Full expressions for the refractive index of the gas [25] and waveguide [26] are computed in the spectral domain, while in the time domain a fourth-order RungeKutta algorithm is used to solve the nonlinear coupled equations, including linear capillary losses. The code calculates frequency generation and back-conversion while accounting for phase and group velocity mismatch effects. All phase-matchable third-order frequency mixing terms are included, as well as self- and cross-phase modulation effects that tend to broaden the pulse spectra. Nonlinear coefficients are available in the literature [27].

For $4 \omega$ generation based on DFFWM $(4 \omega=3 \omega+$ $2 \omega-\omega)$, the predicted phase-matched $\left(\Delta k_{4}=0\right)$ pressures based on this more sophisticated model are 40 and 143 Torr for the two lowest-order modes $\mathrm{EH}_{11}$ and $\mathrm{EH}_{12}$, respectively. This is in agreement with experimental observations and simple calculations. For cascaded process within a single fiber, the generation of light at $4 \omega$ also depends on the phase mismatch in the generation of $3 \omega$. When $\Delta k_{3}+\Delta k_{4}=0$, the field at $3 \omega$ oscillates at the same period as the $4 \omega$ phase mismatch. This corresponds to quasi-phase-matched generation of $4 \omega$ light - the $3 \omega$ pump is present predominantly when it contributes "in phase" to the $4 \omega$ field. Numerical integration confirms that the signal at $4 \omega$ will also peak at pressures of 53 and 129 Torr for the $\mathrm{EH}_{11}$ and $\mathrm{EH}_{12}$ modes, respectively, where $\Delta k_{3}+\Delta k_{4}=0$, in close agreement with Fig. 2(b). In the case of the short fiber, quasi-phase-matched generation is dominant over the direct DFFWM process, since very little pump $3 \omega$ light is available. This is the first observation of such quasi-phase-matching phenomena in the deep-UV region of the spectrum. Our calculations also predict that the $5 \omega$ output depends either on the square of the $3 \omega$ field, or linearly on both the $3 \omega$ and $4 \omega$ fields, depending upon which DFFWM process is giving rise to the signal $(5 \omega=2 \times 3 \omega-\omega$, or $5 \omega=4 \omega+2 \omega-\omega$, or $5 \omega=4 \omega+3 \omega-2 \omega)$. Because the driving fields at $3 \omega$ and $4 \omega$ are being generated within the same one-stage fiber, the $5 \omega$ signal follows the $3 \omega$ and $4 \omega$ signals independent of phase-matching pressure, as observed experimentally.

An interesting question to consider is if perturbation theory is valid at the intensities of $10^{13} \mathrm{~W} \mathrm{~cm}^{-2}$ used in this experiment. The validity of perturbation theory affects most of all how strong the nonlinearity is, and to a lesser extent the phase-matching conditions that are dominated by the linear index of the gas and plasma. Thus, our conclusions regarding the dominance of third-order cascaded processes for efficient, broad-bandwidth, VUV generation are valid.
In conclusion, we have demonstrated, for the first time, macroscopic conversion from the visible into the deep UV using cascaded four-wave mixing processes in a gas. Such cascaded processes exhibit higher efficiencies and broader bandwidths than other schemes for generating ultrashort-pulse light in the deep UV, and will enable many applications in science and technology.

The authors gratefully acknowledge support from the Department of Energy and the National Science Foundation.

*Department of Physics, Colorado School of Mines, Golden, CO.

†Email address: murnane@jila.colorado.edu

[1] C. G. Durfee, S. Backus, M. M. Murnane, and H.C. Kapteyn, Opt. Lett. 22, 1565 (1997).

[2] A. Rundquist et al., Science 280, 1412 (1998).

[3] R. Bartels et al., Nature (London) 406, 164 (2000).

[4] N. A. Anderson et al., Chem. Phys. Lett. 323, 365 (2000).

[5] A. Rettenberger and R. Haight, Surf. Sci. 414, 197 (1998).

[6] S. Lejnine et al., Nucleic Acids Res. 27, 3676 (1999).

[7] T. C. Weinacht, J. Ahn, and P. H. Bucksbaum, Nature (London) 397, 233 (1999).

[8] V. Blanchet, M. Z. Zgierski, T. Seideman, and A. Stolow, Nature (London) 401, 52 (1999).

[9] G. C. Bhar, U. Chatterjee, A. M. Rudra, and P. Kumbhakar, Quantum Electron. 29, 800 (1999).

[10] V. Petrov et al., IEEE J. Sel. Top. Quant. 5, 1532 (1999).

[11] J. Ringling et al., Opt. Lett. 18, 2035 (1993).

[12] R. Mahon, T. J. McIlrath, V.P. Myerscough, and D. W. Koopman, IEEE J. Quantum Electron. 15, 444 (1979).

[13] O. Kittelmann et al., Opt. Lett. 21, 1159 (1996).

[14] J. H. Glownia, D. R. Gnass, and P. P. Sorokin, J. Opt. Soc. Am. B 11, 2427 (1994).

[15] A. J. Merriam et al., Phys. Rev. Lett. 84, 5308 (2000).

[16] C. Dorman, I. Kucukkara, and J. P. Marangos, Phys. Rev. A 61, 013802 (2000).

[17] G. I. Stegeman, D. J. Hagan, and L. Torner, Opt. Quantum Electron. 28, 1691 (1996).

[18] X. D. Mu et al., Opt. Lett. 25, 117 (2000).

[19] V. V. Rostovtseva, A. P. Sukhorukov, V. G. Tunkin, and S. M. Saltiel, Opt. Commun. 22, 56 (1977).

[20] S. A. Akhmanov, V. A. Martynov, S. M. Saltiel, and V. G. Tunkin, JETP Lett. 22, 65 (1975).

[21] A. Goehlich, U. Czarnetzki, and H. F. Dobele, Appl. Opt. 37, 8453 (1998).

[22] H. Crespo, J. T. Mendonca, and A. Dos Santos, Opt. Lett. 25, 829 (2000).

[23] S. Meyer, B. N. Chichkov, and B. Wellegehausen, J. Opt. Soc. Am. B 16, 1587 (1999).

[24] S. Backus, C. Durfee, M. M. Murnane, and H. C. Kapteyn, Rev. Sci. Instrum. 69, 1207 (1998).

[25] P. J. Leonard, At. Data Nucl. Data Tables 14, 21 (1974).

[26] E. A. J. Marcatili and R. A. Schmeltzer, Bell Syst. Tech. J. 43, 1783 (1964).

[27] H. J. Lehmaier, W. Leupacher, and A. Penzkofer, Opt. Commun. 56, 67 (1985). 